\title{
Monitoring the prevalence of methamphetamine-related presentations at psychiatric hospitals in Cape Town, South Africa
}

\author{
A Plüddemann', S Dada1, CDH Parry',2, R Kader', JS Parker³, H Temmingh³, S van Heerden², C de Clercq ${ }^{3}$, Lewis³ \\ ${ }^{1}$ Alcohol \& Drug Abuse Research Unit, Medical Research Council, Tygerberg, South Africa \\ 2Department of Psychiatry, University of Stellenbosch, Tygerberg, South Africa \\ 3Department of Psychiatry \& Mental Health, University of Cape Town, Observatory, South Africa
}

\begin{abstract}
Objective: This study aimed to determine a demographic profile of methamphetamine (MA)-related admissions to major psychiatric services in Cape Town, obtain a substance use profile from admitted patients, a profile of common MA-related symptoms encountered during the assessment of the patients presenting with MA-related problems, and a brief profile of the psychiatric diagnoses made. Method: Staff in six psychiatric hospitals or wards in Cape Town collected data on methamphetamine related admissions between July and December 2008 using a one-page record review form. The data collection form consisted of the patient's demographic details, presenting symptoms, previous admission details, current MA and other substance use information, and DSM-IV diagnosis. Results: A total of 235 forms were completed. Most patients were male (69\%) and the mean age was 25 years. The most common presenting symptoms were aggressive behaviour (74\%), followed by delusions (59\%) and hallucinations (57\%). Males were two times more likely to present with aggression as compared to females, while females were significantly more likely to present with depressed mood or euphoric/elevated mood. The majority of patients had substanceinduced psychotic disorder (41\%), followed by schizophrenia (31\%). Twelve percent (12\%) had bipolar mood disorder. Conclusion: MA-related psychiatric admissions pose serious challenges to all health services dealing with these patients. Further training and treatment protocol development and distribution is indicated.
\end{abstract}

Keywords: Methamphetamine; Admissions; Psychosis; South Africa

Received: 15-11-2011

Accepted: $26-03-2012$

doi: http://dx.doi.org/10.4314/ajpsy.v16il.8

\section{Introduction}

Methamphetamine (MA) is a white and odourless crystalline powder that easily dissolves in water or alcohol. It is a powerful psychoactive stimulant that affects many areas of the central nervous system and it is taken orally, intranasally, intravenously or by smoking. MA normally provides a sense of euphoria and energy through facilitating the release of large amounts of the

\section{Correspondence}

Dr A Plüddemann

Alcohol \& Drug Abuse Research Unit, Medical Research Council

PO Box 19070, Tygerberg 7505, South Africa

email: andreas.pluddemann@mrc.ac.za neurotransmitters dopamine, norephinephrine and ephenephrine from the nucleus accumbens. ${ }^{1}$ Long term effects of MA include early reduction in the density of cortical white matter, reduced glucose metabolism in the thalamus, weight loss, and increased glucose metabolism in the parietal cortex. These brain changes are associated with acute and chronic cognitive impairments and emotional deficits. ${ }^{2,3} \mathrm{MA}$ seemed to emerge in South Africa in 2002 and was most commonly used in Cape Town with the street name "speed".

Data collected by the Medical Research Council from specialist substance abuse treatment centres in Cape Town (as part of the South African Community Epidemiology Network on Drug Use - SACENDU) indicated a sharp increase in the proportion of patients reporting MA as a 
primary substance of abuse. In the first half of 2002 only four of 1608 patients (0.2\%) reported MA as a primary substance of abuse. In 2004, this had increased to $19.3 \%$ and there was extensive community concern around the use of MA (becoming known locally as "tik") in certain suburbs of Cape Town, particularly as a result of the rapid escalation in the use of the drug by adolescents and young adults. In response provincial government appointed a task team in one of the most affected areas to deal with the problem. By 2006, $42 \%$ of patients in specialist substance abuse treatment centres in Cape Town reported MA as their primary substance of abuse, declining slightly by the end of 2008 to 35\%. ${ }^{4}$ A study conducted in 2005 with 4605 high-school learners in Cape Town, investigating the issues of sexual risk behaviour, attitudes and beliefs with regard to HIV and AIDS, revealed that $13 \%$ of the male and $12 \%$ of the female learners had used MA at least once in their life. ${ }^{5}$

MA is known to provide users with pleasurable effects in the beginning, such as high energy levels, selfconfidence, being alert and sociability. ${ }^{6}$ These effects were also revealed in a qualitative study conducted in Thailand on initiation of MA. Drug users reported that MA helped them to enhance productivity at school or at work, and after taking it they would feel like working nonstop. ${ }^{7}$ On the negative side MA use has been found to produce adverse effects such as psychosis, hallucinations, anxiety, and users become violent and depressed which may lead to suicide. ${ }^{8-10} \mathrm{MA}$ also impacts on the health of users as they tend to engage in sexual risk behaviour potentially leading to contracting HIV and other sexually transmitted infections. ${ }^{5}$

While data collected from specialist substance abuse treatment centres in Cape Town has already indicated a sharp escalation in the use of MA, there is currently very limited data available on the extent and impact of MArelated presentations to psychiatric services in Cape Town. This sector is also likely to serve a population which may not have access to specialist substance abuse facilities due to affordability, availability and accessibility issues. Anecdotal evidence suggests that this increased substantially from 2006 and is placing a large burden on psychiatric services in Cape Town.

The present study aimed to determine a demographic profile of MA-related admissions to major psychiatric services in Cape Town, obtain a substance use profile from admitted patients, a profile of common MA-related symptoms encountered during the assessment of the patients presenting with MA-related problems, and a brief profile of the diagnoses made in order to inform mental health services policies and practices.

\section{Methods}

\section{Study design and sampling strategy}

The following public psychiatric hospitals were recruited to participate in a cross-sectional study: Lentegeur Hospital, Stikland Hospital, Valkenberg Hospital, Tygerberg Hospital (Wards D and G lower ground), Karl Bremer Hospital and Groote Schuur Hospital (Ward C23). All patients' records that had a MA-related diagnosis were reviewed as part of this study. Males and females and all age groups were included. Data collection took place from 1 July to 31 December 2008. Records were completed prospectively on all patients admitted consecutively during this period who reported using MA. MA use was thus the primary inclusion criteria for the study.

\section{Procedures}

The data collection form consisted of the patient's demographic details, a check list of presenting symptoms compiled in consultation with psychiatrists (including aggressive behaviour, paranoid thoughts, delusions, hallucinations, thought disordered, depressed mood, euphoric/elevated mood, sleep problems, excessive or uncharacteristic anxiety, and 'other'), previous admission details, current MA and other substance use information, and DSM-IV diagnosis. The clinical diagnoses were made by the treating doctor, supervised by consultant psychiatrists, responsible for the treatment and care of the patients, and in a number of hospitals by multidisciplinary teams. To ensure confidentiality and anonymity, there were no names or personal identifying information recorded on the data collection form. Hospital staff that administered the data collection form were trained in completion of the form. Forms were mostly completed by registrars or medical officers treating the patient (or case managers), under supervision of a consultant psychiatrist in charge of the patient's care, in conjunction with discharge clinical summaries, or on the final clinical impressions of the attendant multidisciplinary team. Completed forms were sent to/or collected by the Medical Research Council staff.

\section{Data analysis}

Data was analysed using the Statistical Package for Social Sciences (SPSS) version 15 and Stata 10. Analysis was mostly descriptive; data was presented using frequency tables, cross-tabulations and summary statistics.

\section{Ethics}

Ethical approval for this study was granted by the Committee for Human Research, Faculty of Health Sciences, University of Stellenbosch and the Research Ethics Committee, Faculty of Health Sciences, University of Cape Town. Permission to conduct the study was also obtained from the various hospital administrators.

\section{Results}

\section{Population profile of patients}

Data was collected from six hospitals and a total of 235 forms were completed on individual patients. The age range of patients was between 15 and 54 years, with a mean age of 25.14 years ( $S D=6.21$ ). The largest group of patients in treatment was in the 20-24 year age-group (42\%) (Table I). Out of 235 patients, 161 patients were males (69\%) and seventy three (31\%) were females. The majority of patients were Coloured (82\%) and a greater proportion of the patients were unemployed (66\%), followed by those working part-time (13\%). Two-thirds had a secondary education or had completed grade 12 and eight patients (4\%) had tertiary education. Almost twothirds of the patients (64\%) reported previous psychiatric admissions. 


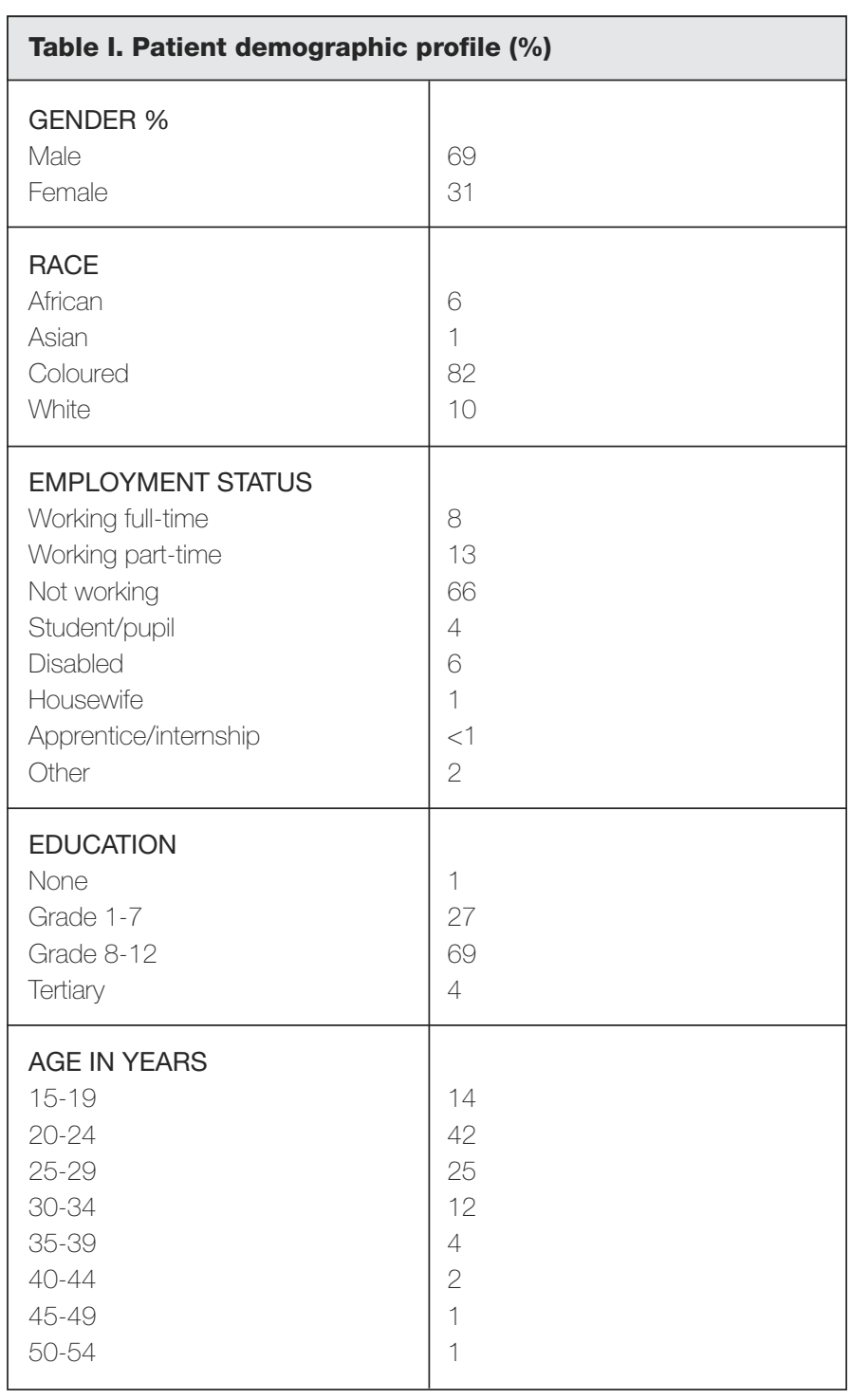

\section{Presenting symptoms}

The majority of patients presented with aggressive behaviour (74\%), followed by delusions (59\%) and hallucinations (57\%). Ninety four patients reported that they had sleeping problems, ninety two had disordered thoughts, and thirty five had depressed mood. Over 90\% of patients presented to hospitals with multiple symptoms. Sixty-four patients presented with three symptoms (27\%), forty eight had four symptoms (20\%) and thirty-five had more than five symptoms (15\%). The most common combination of multiple symptoms were aggressive behaviour/paranoid thoughts/delusions (30\%), aggressive behaviour/paranoid thoughts (10\%), and paranoid thoughts/delusions (7\%). 'Paranoid thought' included paranoid ideas, which could be shifted by rational argument to some degree, and was generally interpreted as sufficient to qualify as a delusion (hence often both were checked on the data collection forms), but not necessarily a qualifier for a diagnosis of psychosis. Some differences across hospitals in always checking both these symptoms on the form may have occurred, depending on interpretation.

Chi-square tests were performed to determine any association between gender and presenting problems. Males were two times more likely to present with aggression as compared to females $(\mathrm{OR}=2.44, \mathrm{df}=1$, $\mathrm{p}=0.004$ ), while females were significantly more likely to present with depressed mood or euphoric/elevated mood (Table II). No significant gender differences were noted for other symptoms.

\section{Primary substance of abuse}

MA was the most common primary substance of abuse in 59\% of admissions, followed by cannabis (32\%) (Table III). This shows that MA was not always necessarily the 'primary substance' (or most frequently used) by the patient, with almost $40 \%$ reporting other substances as their primary substance of abuse, most notably cannabis. Of those patients who reported MA as their most frequently used substance, the majority reported that they smoked the drug (96\%). Thirty-one percent of patients reported that they used MA daily, and a further third used it 2-6 days per week in the past month. Almost 20\%, however, reported using it fairly infrequently (once a week or less) and a further 16\% reported that they had not used it in the past month. A number of these patients (who claimed not to have used MA in the past month) were however diagnosed with a substance-induced psychotic disorder.

\section{Table II. Presenting symptoms by gender}

\begin{tabular}{|c|c|c|c|c|c|c|}
\hline \multirow[t]{2}{*}{ Symptom } & \multicolumn{2}{|c|}{ Males } & \multicolumn{2}{|c|}{ Females } & \multirow[t]{2}{*}{ Odds Ratio } & \multirow[t]{2}{*}{$P$-value } \\
\hline & $n$ & $\%$ & $n$ & $\%$ & & \\
\hline $\begin{array}{l}\text { Aggressive behaviour } \\
\text { Paranoid thoughts } \\
\text { Delusions } \\
\text { Hallucinations } \\
\text { Thought disordered } \\
\text { Depressed mood } \\
\text { Euphoric/elevated mood } \\
\text { Sleep problems } \\
\text { Anxiety }\end{array}$ & $\begin{array}{l}129 \\
83 \\
90 \\
88 \\
58 \\
13 \\
35 \\
60 \\
16\end{array}$ & $\begin{array}{l}81 \\
52 \\
56 \\
55 \\
36 \\
8 \\
22 \\
38 \\
10\end{array}$ & $\begin{array}{l}46 \\
42 \\
46 \\
48 \\
33 \\
20 \\
30 \\
29 \\
5\end{array}$ & $\begin{array}{l}63 \\
58 \\
63 \\
66 \\
45 \\
27 \\
41 \\
40 \\
7\end{array}$ & $\begin{array}{l}2.44 \\
0.79 \\
0.76 \\
0.64 \\
0.69 \\
0.23 \\
0.40 \\
0.91 \\
1.51\end{array}$ & $\begin{array}{l}0.004^{*} \\
0.422 \\
0.331 \\
0.122 \\
0.194 \\
0.000^{\star} \\
0.002^{\star} \\
0.746 \\
0.436\end{array}$ \\
\hline
\end{tabular}


Table III: Proportions of patients reporting various substances as primary, secondary or tertiary substances of abuse

\begin{tabular}{|c|c|c|c|c|c|c|}
\hline & \multicolumn{2}{|c|}{ Primary substance } & \multicolumn{2}{|c|}{ Secondary substance* } & \multicolumn{2}{|c|}{ Tertiary substance* } \\
\hline & $n$ & $\%$ & $n$ & $\%$ & $n$ & $\%$ \\
\hline Alcohol & 9 & 4 & 9 & 4 & 26 & 11 \\
\hline Cocaine/Crack & 2 & 1 & 5 & 2 & 6 & 3 \\
\hline Cannabis (Dagga) & 75 & 32 & 73 & 31 & 19 & 8 \\
\hline Heroin & 2 & 1 & 1 & $<1$ & 3 & 1 \\
\hline Mandrax & 5 & 2 & 23 & 10 & 23 & 10 \\
\hline Other & 1 & $<1$ & 3 & 1 & 6 & 3 \\
\hline MA ('Tik') & 139 & 59 & 65 & 28 & 17 & 7 \\
\hline MA \& heroin & 1 & $<1$ & - & - & - & - \\
\hline TOTAL & \multicolumn{2}{|l|}{234} & \multicolumn{2}{|l|}{182} & \multicolumn{2}{|l|}{104} \\
\hline
\end{tabular}

Cannabis was the most common secondary substance of abuse, reported by $31 \%$ of all patients, however MA was also a secondary substance in a number of cases (28\%), and in some cases only a tertiary substance (7\%). Alcohol and Mandrax were mostly reported as the third substance of abuse, by $11 \%$ and $10 \%$ of all patients respectively (Table III). Few patients reported heroin use, although one patient reported both MA and heroin as primary substances of use.

\section{Clinical disorders: DSM-IV Axis I}

The majority of the 235 patients had substance-induced psychotic disorder (41\%), followed by schizophrenia (31\%). Twelve per cent had bipolar mood disorder. A large variety of different combinations of these disorders, together with substance use disorders and other disorders were recorded. Other disorders that were diagnosed included dementia, major depression episodes and anxiety disorders. Two patients presented with methamphetamine intoxication only.

\section{Personality disorders: DSM-IV Axis II}

Diagnosis on Axis II was deferred for almost half the patients (49\%), about 5\% had antisocial personal traits or cluster B personality traits (3\%). About $2 \%$ of patients had borderline to moderate intellectual disability and $<1 \%$ had histrionic and schizoid traits. These disorders were probably underdiagnosed due to the difficulty in making a diagnosis of a personality disorder in the presence of Axis 1 pathology.

\section{Psychosocial and environmental problems: DSM-IV Axis IV}

The majority of patients were unemployed (66\%) (Table I) and about $1 \%$ had unstable or ad hoc jobs. Patients also reported having conflicts at home with parents, relatives and family in general (12\%). Family problems included having family members who also abused substances (and this caused problems at home), having family with history of mental illness and multiple disabilities, parents with depression and poor relationships within families. This was reported by $11 \%$ of patients. Some patients also reported lacking social support (9\%), living on the streets or poor conditions, living in communities with gangs and neighborhoods that sell drugs.

\section{Discussion}

MA is a major problem in Cape Town, but little attention has been given to psychiatric problems experienced by users. This study represents a first attempt at trying to determine the number of MA-related presentations to psychiatric facilities in Cape Town, and it highlights the significant burden that MA-related psychiatric problems, particularly MA-induced psychosis, are placing on psychiatric services. MA users tend to be agitated, aggressive and they experience severe psychotic symptoms; this indicates the challenges of treating these patients. The initial management and "containment" of these patients poses a challenge to hospital staff, particularly in hospitals with a relatively small capacity for psychiatric intervention/services and due to high staff turnover and rotation. The drugs' side effects also make psychotherapeutic intervention challenging, as there may be problems with concentration and memory (retention) for some time after initial presentation. A review of record admissions conducted at an acute psychiatric admissions ward at one hospital in Cape Town (Tygerberg) over a six month period in 2006, compared with the same period in 2002 , indicated that no patients reported MA use in 2002 , whereas 37\% reported MA use in 2006 and that most of these patients (67\%) were adolescents. ${ }^{11}$

Studies in other countries have indicated that up to almost a third of methamphetamine users experience psychosis at some stage of their use, particularly regular users. ${ }^{12,13}$ Surveys and data collected from treatment centres in Cape Town (referred to in the introduction) would thus suggest that potentially thousands of methamphetamine users may require psychiatric treatment while using methamphetamine. Anecdotally, and as indicated to some extent by this report, many of these patients will return for psychiatric care, as they may start using MA again and therefore again experience psychotic episodes. These psychotic symptoms tend to take longer to treat (and to subside) with each re-admission. MA use is 
also associated with antisocial behaviour as well as sexual risk behaviour. 5,12 MA users tend to engage in unsafe sex with anonymous partners, are more likely to exchange sex for money or drugs and they experience trauma from prolonged intercourse and failure to use condoms while high, increasing the risk of exposure to the HI virus. ${ }^{14}$ This may further complicate the treatment and management of the patient.

An interesting finding was that female patients were significantly more likely to present with either depressed or elevated mood than male patients. Conversely, male patients were more likely to show signs of aggression than female patients. This may indicate real differences in effects of the drug on males and females or the effect of social expectations and will have implications for treatment. A review of gender differences in methamphetamine use and responses found that MA use was more often associated with depression in females. The authors, however, hypothesized that females may more often use MA to self-medicate pre-existing depression. ${ }^{15}$

\section{Study limitations}

Unfortunately not all hospitals collected data as planned. One additional hospital did not collect data between July and December 2008, despite an indication of willingness to do so. However, some data collection commenced in January 2009 and discussions with staff indicated it to have perhaps the highest number of acute MA-related psychiatric presentations in the city. Two of the six hospitals collected data three months after the commencement of the study due to a delay in obtaining ethical approval from the University of Cape Town. The number of patients recorded in this project is thus likely to be a significant underestimate of the actual number that were admitted for treatment. It was also not possible to determine what proportion of total psychiatric admissions were patients who used MA, as hospitals did not collect this data. However, Lentegeur hospital reported that $16 \%$ of all acute admissions in the 12 months up to April 2008 were MA-associated admissions.

\section{Conclusion}

The demand and need for psychiatric intervention related to MA use is thus likely to be far greater than can be measured by admissions to existing psychiatric (and even generalist substance abuse) services. However there are strong indications that the demand for services far outweighs the availability of services currently. Clearly there are major challenges in how to provide the necessary training and support to all health services in dealing with the challenges these patients pose, and further training and treatment protocol development and distribution is indicated.

Suggestions for future research include investigating differences in duration of methamphetamine use on psychiatric symptoms and the implications for treatment. Effects of different combinations of drug use (e.g. methamphetamine and heroin) on mental health and psychiatric problems also remain underresearched.

\section{Acknowledgments}

The authors would like to acknowledge the support of hospital staff in completing the data collection forms for this project and the funder of the project, the National Department of Health: Mental Health \& Substance Abuse Directorate.

\section{References}

1. McCann UD, Ricaurte GA. Amphetamine neurotoxicity: accomplishments and remaining challenges. Neuroscience and Biobehavioural Reviews 2004; 27:821-6.

2. Brecht ML, O'Brien A, von Mayrhauser C, Anglin MD. Methamphetamine use behaviors and gender differences. Addict Behav 2004; 29(1):89-106.

3. Cretzmeyer M, Sarrazin MV, Huber DL, Block RI, Hall JA. Treatment of methamphetamine abuse: research findings and clinical directions. J Subst Abuse Treat 2003; 24(3):267-77.

4. Pluddemann A, Myers BJ, Parry CD. Surge in treatment admissions related to methamphetamine use in Cape Town, South Africa: implications for public health. Drug Alcohol Rev 2008; 27(2): 185-9.

5. Pluddemann A, Flisher AJ, Mathews C, Carney T, Lombard C. Adolescent methamphetamine use and sexual risk behaviour in secondary school students in Cape Town, South Africa. Drug Alcohol Rev 2008; 27(6):687-92.

6. Saltman DC, Newman CE, Mao L, Kippax SC, Kidd MR. Experiences in managing problematic crystal methamphetamine use and associated depression in gay men and HIV positive men: in-depth interviews with general practitioners in Sydney, Australia. BMC Fam Pract 2008; 9:45.

7. Sherman SG, Gann D, German D, Sirirojn B, Thompson $N$, Aramrattana A, et al. A qualitative study of sexual behaviours among methamphetamine users in Chiang Mai, Thailand: a typology of risk. Drug \& Alcohol Review 2008; 27(3):263-9.

8. Zweben JE, Cohen JB, Christian D, Galloway GP, Salinardi M, Parent D, et al. Psychiatric symptoms in methamphetamine users. The American Journal on Addictions 2004; 13:181-90.

9. McGregor C, Srisurapanont M, Jittiwutikarn J, Laobhripatr S, Wongtan T, White JM. The nature, time course and severity of methamphetamine withdrawal. Addiction 2005; 100(9):1320-9.

10. Glasner-Edwards S, Mooney LJ, Marinelli-Casey P, Hillhouse M, Ang A, Rawson R. Identifying methamphetamine users at risk for major depressive disorder: findings from the methamphetamine treatment project at three-year follow-up. Am J Addict 2008; 17(2):99-102.

11. Vos PJ, Cloete KJ, le Roux A, Kidd M, Jordaan GP. A retrospective review of trends and clinical characteristics of methamphetamine-related acute psychiatric admissions in a South African context. Afr J Psychiatry 2010; 13(5):390-4.

12. Darke S, Kaye S, McKetin R, Duflou J. Major physical and psychological harms of methamphetamine use. Drug Alcohol Rev 2008; 27(3):253-62.

13. Salo R, Flower K, Kielstein A, Leamon MH, Nordahl TE, Galloway GP. Psychiatric comorbidity in methamphetamine dependence. Psychiatry Res 2011; 186(2-3):356-61.

14. German D, Sherman SG, Latkin CA, Sirirojn B, Thomson N, Sutcliffe CG, et al. Young Thai women who use methamphetamine: intersection of sexual partnerships, drug use, and social networks. Int J Drug Policy 2008; 19(2):122-9.

15. Dluzen DE, Liu B. Gender differences in methamphetamine use and responses: a review. Gend Med 2008; 5(1):24-35. 